$\mathrm{DE}$

M E D I C I N A

T R O P I C A L

$\mathrm{DE}$

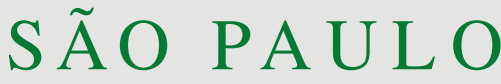

JOURNAL OF THE SÃO PAULO INSTITUTE OF TROPICAL MEDICINE

${ }^{1}$ Universidade Federal do Rio Grande do Norte, Departamento de Infectologia, Natal, Rio Grande do Norte, Brazil

2Universidade de São Paulo, Faculdade de Medicina, Hospital das Clínicas, Divisão Laboratório Central, São Paulo, São Paulo, Brazil

${ }^{3}$ Centro de Patologia Clínica, Laboratório de Microbiologia, Natal, Rio Grande do Norte, Brazil

Correspondence to: Hareton Teixeira Vechi Universidade Federal do Rio Grande do Norte, Departamento de Infectologia, Rua Cônego Monte, $n^{\circ} 110$, CEP 59037-170, Natal, RN, Brazil

Tel.: +55 84 99178-1695

Fax: $+55843215-1603$.

E-mail: haretonvechi@gmail.com

Received: 26 September 2018

Accepted: 24 October 2018

\section{Chronic cavitary pneumonia by Rhodococcus equi in a highly prevalent tuberculosis country: a diagnosis challenge}

\author{
Hareton Teixeira Vechi' ${ }^{1}$, Eduardo Teodoro Gurgel de Oliveira1, Marise Reis de \\ Freitas ${ }^{1}$, Flávia Rossi ${ }^{2}$, Maria Helena Marques Fonseca de Britto ${ }^{3}$, Manoella \\ do Monte Alves ${ }^{1}$
}

\section{ABSTRACT}

Rhodococcus equi is a facultative aerobic, intracellular, non-motile, non-spore-forming, Gram-positive, weakly acid-fast coccobacillus belonging to the group of nocardioform actinomycetes. $R$. equi infections are rare opportunistic illnesses in patients with Acquired Immunodeficiency Syndrome (AIDS), associated with a high mortality rate. The most common clinical presentation of $R$. equi infections is a chronic cavitary pneumonia. Due to its acid-fastness, $R$. equi can be mistaken for others acid-fast organisms, as Mycobacterium tuberculosis. In turn, $R$. equi is also a gram-positive pleomorphic bacteria and can be mistaken for diphtheroids or Micrococcus organisms, being accidentally disregarded as oral contaminants in sputum cultures. Therefore, in Brazil, a highly prevalent tuberculosis (TB) country, pulmonary infections caused by $R$. equi may mimic pulmonary TB and represent a diagnostic challenge. Here, we report on a case of chronic cavitary pneumonia by $R$. equi in a Human Immunodeficiency Virus (HIV)-infected patient, focusing on diagnostic aspects.

KEYWORDS: Rhodococcus equi. AIDS. HIV. Tuberculosis. Necrotizing pneumonia.

\section{INTRODUCTION}

Rhodococcus equi is a facultative aerobic, intracellular, non-motile, non-sporeforming, Gram-positive, weakly acid-fast coccobacillus belonging to the group of nocardioform actinomycetes ${ }^{1} . R$. equi is a pathogen of well-recognized relevance in veterinary medicine, causing lung abscess in foals and submaxillary adenitis in swine ${ }^{2}$. Since the first report of human infection in $1967^{3}, R$. equi has been appreciated as an opportunistic pathogen, especially in patients with impaired immune system such as organ solid and hematopoietic stem cells recipients and HIV-infected patients ${ }^{4}$.

Due to AIDS epidemic, the human infections by $R$. equi are increasing in frequency ${ }^{1,4}$. Most of $R$. equi infections occur in HIV-infected patients with a CD4 T lymphocytes count below 200 cells $/ \mu \mathrm{L}$ and the most common clinical presentation is a chronic cavitary pneumonia, similar to foals ${ }^{2,5}$. Extrapulmonary disease, with or without concomitant pulmonary infection, can also occur and affects mainly central nervous system as well as skin and soft tissues ${ }^{5}$. The $R$. equi disease has a high mortality rate in AIDS patients. Besides a prolonged combination antibiotic therapy, antiretroviral therapy (ART) is also important and associated with higher survival rates in HIV-infected patients ${ }^{5,6}$.

Here, we report on a case of a chronic cavitary pneumonia by Rhodococcus equi in a HIV-infected patient, focusing on diagnostic aspects. 


\section{CASE REPORT}

A 45-year-old HIV-infected man, a hairdresser, sought our emergency department on May 2017 complaining of productive cough for 7 months, with a purulent sputum, associated with a pleuritic chest pain on the right side. During this time, the patient reported high intensity fever (axillary temperature of $39^{\circ} \mathrm{C}$ ) accompanied by anorexia, asthenia and weight loss of $5 \mathrm{~kg}$. Over the last month, he also referred progressive dyspnea on exertion. The patient denied other symptoms.

The HIV infection has been diagnosed 4 years ago and his current ART consisted of efavirenz, zidovudine and lamivudine. His medical history was remarkable for the poor adherence to ART in the last 2 years. Recent CD4 and CD8 T lymphocytes counts were 45 (5.0\%) and $517(57.0 \%)$ cells $/ \mu \mathrm{L}$ respectively, and the viral load was 199,233 copies/mL (5.299 $\left.\log _{10}\right)$. He had previous hospitalizations for cerebral toxoplasmosis on 2013, his AIDS-defining opportunistic illness, and retinal detachment secondary to cytomegalovirus retinitis on July 2016. His mother had pulmonary tuberculosis 30 years ago. The patient used to drink fermented beverages occasionally and smoke almost a cigarette pack daily. He lived in the outskirts of Natal, the capital of Rio Grande do Norte State, Brazil, and raised a cat, a dog, chickens and a horse in the backyard.
On admission, vital signs were axillary temperature $38.4{ }^{\circ} \mathrm{C}$, blood pressure $100 / 60 \mathrm{mmHg}$, pulse rate $99 \mathrm{bpm}$ and respiratory rate $25 / \mathrm{min}$. The physical examination was remarkable for the presence of oropharyngeal candidiasis and diminished vesicular breath sounds with inspiratory crackles heard in the area of right mid and lower lung fields. Laboratory tests initially showed: hemoglobin of $10.8 \mathrm{~g} / \mathrm{L}$, white cells count of $5.7 \times 10^{9} / \mathrm{L}$ ( $76 \%$ neutrophils and $18 \%$ lymphocytes) and platelet counts of $134 \times 10^{9} / \mathrm{L}$. The kidney and liver function tests were normal. The serum lactate dehydrogenase level was also normal. Chest $\mathrm{X}$-ray showed an area of consolidation with a thick walled cavitation containing an air-fluid level on the right mid lung field (Figure 1A). Computed tomography of the chest showed a pleuropulmonary fluid-gas collection with thickened walls and irregular edges, located in the anterior portion of the middle third of the right hemithorax, with an estimated volume of $288 \mathrm{~mL}$ (Figure 1B). Blood cultures were negative for bacteria and fungi.

The patient's sputum samples were collected. An acid-fast bacilli smear of the specimen was negative on Ziehl-Neelsen stain, but showed coccobacillary organisms on modified Kinyoun stain (Figure 2A). The GeneXpert MTB/RIF sputum test was negative. Then, the specimen was inoculated on 5\% sheep blood agar, chocolate agar and MacConkey's agar plates incubated for $48 \mathrm{~h}$ at $37^{\circ} \mathrm{C}$. The sputum sample was also treated by $\mathrm{N}$-acetyl

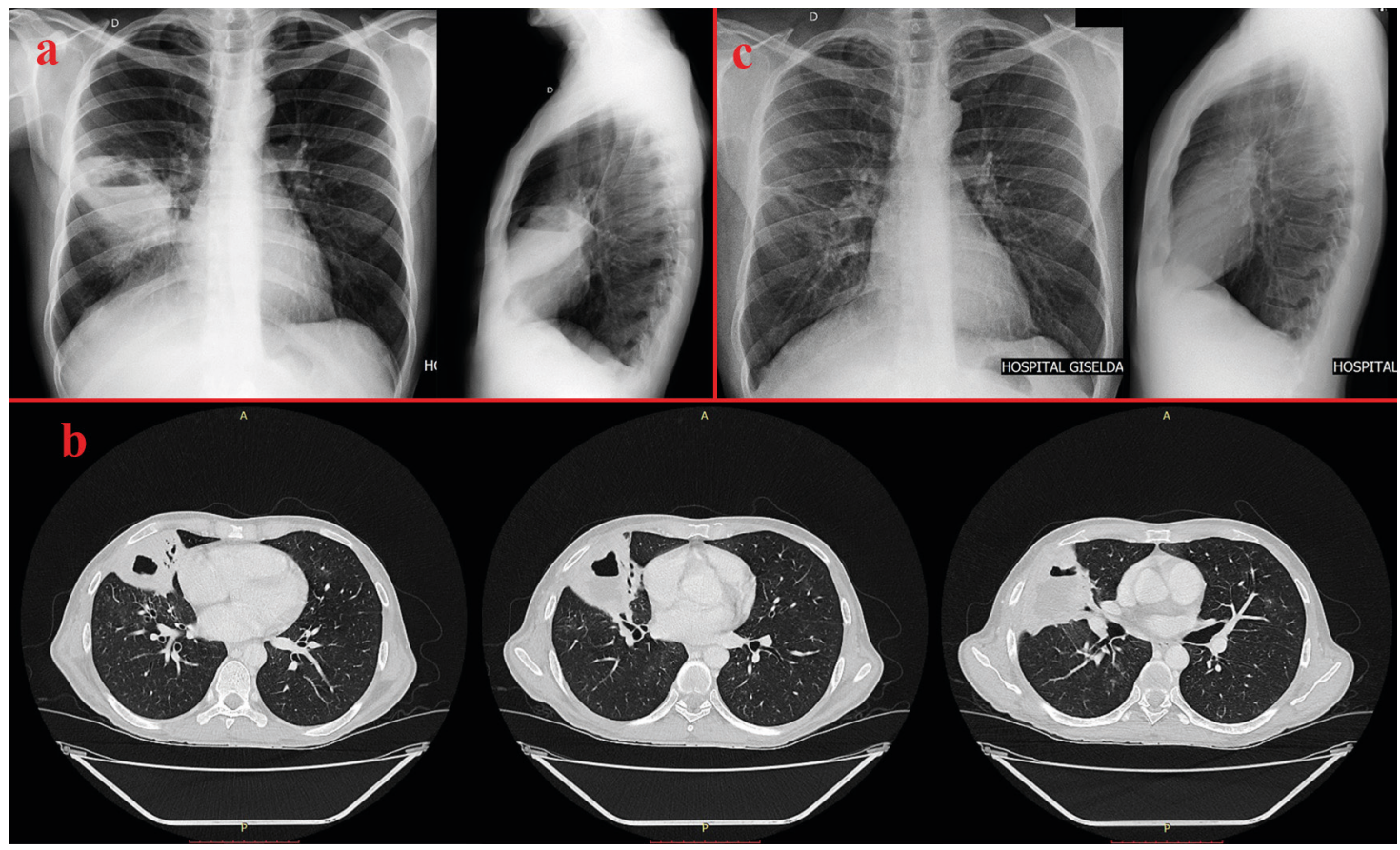

Figure 1 - A) Chest X-ray showing a consolidation area with a thick -walled cavitation containing an air-fluid level in the right mid lung field on the patient's admission; B) Computed tomography showing a pleuropulmonary fluid-gas collection located in the right middle lung lobe; C) Chest X-ray showing linear fibrotic bands with cicatricial appearance in the right mid lung field four months after treatment for Rhodococcus equi pneumonia. 
L-cysteine sodium hydroxide method, inoculated on slopes of Lowenstein-Jensen medium and incubated for 8 weeks at $37^{\circ} \mathrm{C}$ in 5-10\% $\mathrm{CO}_{2}$. Pink colored and smooth mucoid bacteria colonies grown on sheep blood and chocolate agar (Figure 2B), but not on MacConkey's agar. The direct microscopic examination on Gram stain showed Gram-positive coccobacilli (Figure 2C) and acid-fast on Kinyoun stain. The mycobacterial culture was negative. The isolate was identified by Vitek ${ }^{\circledR} 2$ automated system (software version 7.01) as Kocuria rosea (95\% probability) using Vitek 2 GP ID card. However, Kocuria rosea is not an acid-fast organism and it is not implicated in pulmonary infections. Considering the possibility of misidentification of the isolate by Vitek ${ }^{\circledR} 2$, the strain was sent to the Microbiology Laboratory of Hospital das Clínicas, São Paulo, Brazil for identification by using matrix-assisted laser desorption ionization-time of flight (MALDI-TOF) Vitek ${ }^{\circledast}$ mass spectrometry (MS). It identified the patient's isolate as Rhodococcus equi with 93\% probability (Figure 2D).

The patient was treated for chronic necrotizing pneumonia by Rhodococcus equi with amikacin at a dose of $1 \mathrm{~g}$ /day intravenously (IV), imipenem at $500 \mathrm{mg}$ q6h IV and moxifloxacin $400 \mathrm{mg}$ daily IV for 2 months at inpatient treatment. After that, he took orally an outpatient combination therapy with levofloxacin at $500 \mathrm{mg}$ daily, trimethoprim-sulfamethoxazole $160 / 800 \mathrm{mg} \mathrm{q12h}$ and azithromycin $500 \mathrm{mg}$ daily for 4 months. The patient had an excellent response to treatment and the symptoms disappeared completely. Chest X-ray performed four months after treatment showed fibrotic changes in middle zone of the right lung (Figure 1C). The antiretroviral therapy has switched for dolutegravir at $50 \mathrm{mg}$ daily combined with ritonavir-boosted darunavir at $600 \mathrm{mg} \mathrm{q12h}$ after genotypic resistance assay results. The CD4 lymphocyte count increased to 236 cells/ $\mu \mathrm{L}$ and HIV viral load was undetectable. The patient did not develop the immune reconstitution inflammatory syndrome nor the relapse of the disease. After treatment, he kept using a trimethoprimsulfamethoxazole double-strength tablet for prophylaxis of R. equi infection and Pneumocystis jirovecii pneumonia.

\section{DISCUSSION}

AIDS is the major predisposing immunosuppressive condition for $R$. equi infections accounting for about two-thirds of cases ${ }^{7}$. In HIV-infected patients, pulmonary

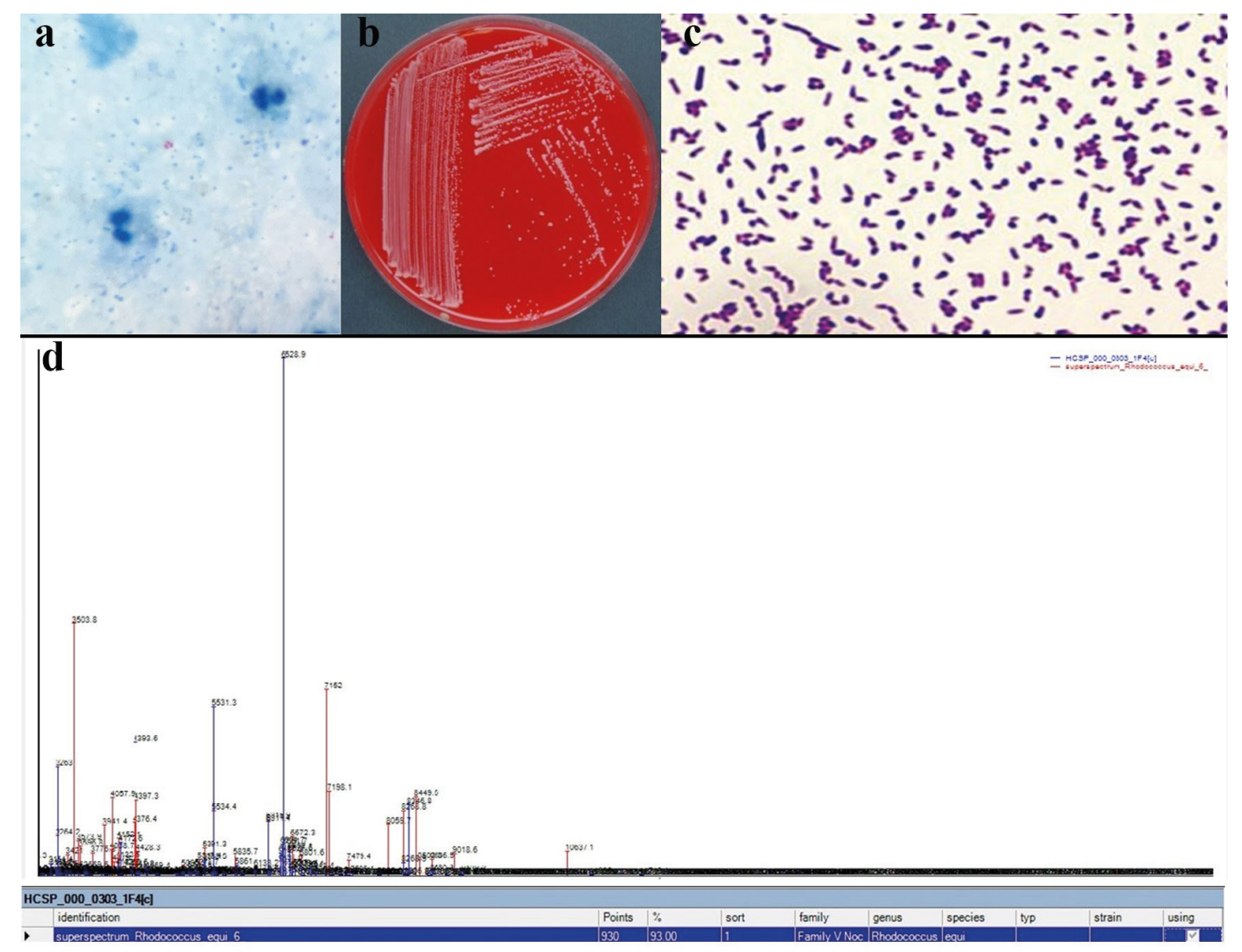

Figure 2 - A) Acid-fast coccobacillary organisms in modified Kinyoun stain; B) Smooth mucoid pink bacteria colonies yielded on $5 \%$ sheep blood agar plate; C) Gram-positive coccobacillus with diphtheroid-like morphology in Gram stain; D) Rhodococcus equi spectral signature from Vitek ${ }^{\circledR}$ MS with Saramis ${ }^{\circledR}$ Software. 
infections are also the most common clinical presentation of R. equi infections, observed in more than $80 \%$ of cases, and present usually as a subacute or chronic necrotizing pneumonia ${ }^{5,8}$. Compared with non-HIV-infected individuals, the symptoms of $R$. equi pneumonia are similar in AIDS patients, but more cases of extrapulmonary disease, with or without concurrent pneumonia are found in the latter group ${ }^{7,9}$.

The soil and gut of herbivorous animals are the natural reservoirs of $R$. equi and the bacterium meets optimal conditions for growth in horse manure ${ }^{2,7,9}$. The high frequency of pulmonary infections in immunocompromised humans indicates that the main mode of $R$. equi acquisition is the inhalation of contaminated material from the soil ${ }^{7,8}$. Takai et $a l .^{10}$ reported that the number of $R$. equi isolated from the air on horse farms increased on warmer, dry and windy days, suggesting aerosolization of soil particles contaminated with $R$. equi as a transmission mechanism.

In HIV- and non-HIV-infected patients with pulmonary disease by R. equi, areas of consolidation with cavitation are the most common radiological features ${ }^{5,6,9,11,12}$. Cavitation is found in more than $75 \%$ of cases and lung abnormalities are predominantly located in the upper lobes $(55 \%)^{11,12}$. Similar to other HIV-infected patients, our patient had Rhodococcus equi pneumonia with cavitation, even though he was severely immunosuppressed, suggesting a direct action of the bacterium causing lung lesions, and not by the host's immune response.

In fact, the pathogenic potential of $R$. equi is associated with its ability to survive inside macrophages and destroy them $^{2}$. The bacterium evades intracellular killing by interfering with the phagosome-lysosome fusion, by the cell wall mycolic acids and by a surface lipoprotein named Virulence-associated protein (Vap). These factors play a relevant role in this survival mechanism ${ }^{2,7,13}$. In addition, $R$. equi induces a non-specific degranulation of infected macrophages, contributing to tissue destruction and neutrophil influx ${ }^{2}$.

In this case, the patient has raised a horse in backyard. This was probably the epidemiological clue for the diagnosis of R. equi pneumonia. However, a history of contact with farm animals, herbivorous manure or occupational exposure is not always present on reports ${ }^{6,8,9}$. One explanation is that this type of information is not actively sought in clinical practice and most of studies about $R$. equi infections are retrospective.

When $R$. equi infection is suspected, the medical staff should explicitly inform this possibility to clinical microbiologists for the following reasons. Firstly, $R$. equi is a pleomorphic bacterium whose morphology varies from coccoid to bacillary depending on the specimen type, growth conditions and type of medium, and it may be mistaken for oral cavity contaminants as diphtheroid

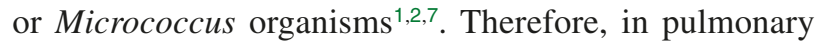
infections, $R$. equi might be present in sputum culture mixed with other pathogens, or even in pure culture, and be disregarded due to the diphtheroid aspect.

Secondly, it is important to choose a reliable identification method for $R$. equi. Conventional methods, based on laboratory bench biochemical tests, can be lengthy and laborious to be performed in a clinical laboratory routine ${ }^{2,14}$. In turn, automated methods would come to solve these negative points. Nevertheless, misidentification by automated systems could occur with a potential negative impact on diagnosis and treatment of the patient, as we observed in this case.

The automated system Vitek ${ }^{\circledR} 2$ misidentified $R$. equi isolate as Kocuria rosea with $95 \%$ probability (very good). In fact, Vitek ${ }^{\otimes}$ identification cards are not capable to identify correctly R. equi isolates ${ }^{15}$ and this is a method limitation. Interestingly, $K$. rosea is a member of the Micrococcaceae family, which also includes Micrococcus species ${ }^{16}$, with which $R$. equi has already been mistaken ${ }^{7}$. K. rosea is part of the oral cavity microbiota and is not implicated in pulmonary infections, even in immunosuppressed patients ${ }^{16}$. In addition, $K$. rosea is not an acid-fast organism and this was the clue to the problem of misidentification.

MALDI-TOF mass spectrometry has proved to be a reliable method for identification of $R$. equi isolates with a rapid turn-around time ${ }^{17}$. We believe MALDI-TOF might be advantageous in the context of R. equi infections due to its ability to diagnose early and accurately, allowing immediate treatment of an infection with high mortality rates in immunosuppressed patients, especially in HIV-infected individuals 5 5,6,8,9.

The third reason is that $R$. equi is an acid-fast organism due to presence of mycolic acids in its cell wall. That is why R. equi may be mistaken for other acid-fast pathogens, especially Mycobacterium tuberculosis ${ }^{2,4,7}$. Nevertheless, unlike M. tuberculosis, R. equi is weakly acid-fast and nonalcohol fast ${ }^{18}$. In this case, acid-fast testing was negative in Ziehl-Neelsen stain and positive in modified Kinyoun stain. This illustrates the importance of using other modified acid-fast techniques in suspected $R$. equi infections cases, as Kinyoun staining, to reveal $R$. equi acid-fastness, because Kinyoun stain is based on an aqueous solution of a weak acid, $1 \%$ sulfuric acid, to promote the discoloration ${ }^{1,18}$.

Our patient lives in Brazil, a country with a high burden of tuberculosis (TB) where current data estimate an incidence of 42 cases per 100,000 inhabitants, and a HIV infection prevalence in incident TB cases of $13 \%{ }^{19}$. Classically, pulmonary TB presents as chronic cavitary disease. It is also known that a positive acid-fast sputum smear often leads to anti-tuberculosis treatment initiation 
in limited resources settings ${ }^{19}$. However, this is the typical presentation in immunocompetent individuals. In HIV-infected patients with CD4 lymphocyte counts below 200 cels $/ \mu \mathrm{L}$, pulmonary TB usually has no cavitation and a lower frequency of positive sputum smears ${ }^{20}$.

Therefore, on clinical and radiographic grounds, R. equi pulmonary infections can mimicry pulmonary tuberculosis, but should be considered more likely as the cause of cavitary pneumonia in HIV-infected patients with severe immunosuppression than M. tuberculosis mostly when: 1) there is a negative acid-fast sputum smear and only ZiehlNeelsen staining was used; 2) a modified acid-fast staining shows the presence of acid-fast cocobacillary bacteria; 3 ) history of contact with farm animals or herbivorous manure or occupational exposure is present.

\section{CONCLUSION}

R. equi infection is a rare opportunistic illness in patients with AIDS and usually presents as pulmonary infections. $R$. equi should be included in the differential diagnosis of chronic cavitary pneumonia in HIV-infected patients with severe immunosuppression, especially in the presence of epidemiological history of contact with farm animals or herbivorous manure. In limited resources settings, when a gram-positive coccobacillus is observed in sputum smears and it is positive in an acid-fast staining, we are presumptively facing a $R$. equi isolate, taking into account the characteristic clinical-epidemiological context.

\section{ACKNOWLEDGMENTS}

We would like to thank the Hospital Giselda Trigueiro for technical assistance.

\section{CONSENT FOR PUBLICATION}

A written informed consent was obtained from the patient for publication of this case report and any accompanying images. Available on request.

\section{CONFLICT OF INTERESTS}

The authors declare that they have no conflict of interests.

\section{FUNDING}

This research did not receive any specific grant from funding agencies of public, commercial, or not-for-profit sectors.

\section{AUTHORS' CONTRIBUTIONS}

HTV was one of the patient's physician assistant and the major contributor in the writing of this manuscript; ETGO was one of the patient's assistant physician and also contributed to the writing of the manuscript; MRF and MMA contributed writing and reviewing the manuscript; MHMFB performed the microbiologic study; FR performed the identification of the patient's isolate. All authors read and approved the final manuscript.

\section{REFERENCES}

1. Scott MA, Graham BS, Verrall R, Dixon R, Schaffner W, Tham KT. Rhodococcus equi: an increasingly recognized opportunistic pathogen. Report of 12 cases and review of 65 cases in the literature. Am J Clin Pathol. 1995;103: 649-55.

2. Prescott JF. Rhodococcus equi: an animal and human pathogen. Clin Microbiol Rev. 1991;4:20-34.

3. Golub B, Falk G, Spink WW. Lung abscess due to Corynebacterium equi. Report of first human infection. Ann Intern Med. 1967;66:1174-7.

4. Verville TD, Huycke MM, Greenfield RA, Fine DP, Kuhls TL, Slater LN. Rhodococcus equi infections of humans. 12 cases and a review of the literature. Medicine (Baltimore). 1994;73:119-32.

5. Torres-Tortosa M, Arrizabalaga J, Villanueva JL, Gálvez J, Leyes M, Valencia ME, et al. Prognosis and clinical evaluation of infection caused by Rhodococcus equi in HIV-infected patients: a multicenter study of 67 cases. Chest. 2003;123:1970-6.

6. Arlotti M, Zoboli G, Moscatelli GL, Magnani G, Maserati R, Borghi V, et al. Rhodococcus equi infection in HIV-positive subjects: a retrospective analysis of 24 cases. Scand J Infect Dis. 1996;28:463-7.

7. Weinstock DM, Brown AE. Rhodococcus equi: an emerging pathogen. Clin Infect Dis. 2002;34:1379-85.

8. Donisi A, Suardi MG, Casari S, Longo M, Cadeo GP, Carosi G. Rhodococcus equi infection in HIV-infected patients. AIDS. 1996;10:359-62.

9. Harvey RL, Sunstrum JC. Rhodococcus equi infection in patients with and without human immunodeficiency virus infection. Rev Infect Dis. 1991;13:139-45.

10. Takai S, Fujimori T, Katsuzaki K, Tsubaki S. Ecology of Rhodococcus equi in horses and their environment on horsebreeding farms. Vet Microbiol. 1987;14:233-9.

11. Muntaner L, Leyes M, Payeras A, Herrera M, Gutierrez A. Radiologic features of Rhodococcus equi pneumonia in AIDS. Eur J Radiol. 1997;24:66-70.

12. Marchiori E, Mendonça RG, Capone D, Cerqueira EM, Souza Júnior AS, Zanetti G, et al. Rhodococcus equi infection in 
acquired immunodeficiency syndrome. Computed tomography aspects. J Bras Pneumol. 2006;32:405-9.

13. Rofe AP, Davis LJ, Whittingham JL, Latimer-Bowman EC, Wilkinson AJ, Pryor PR. The Rhodococcus equi virulence protein VapA disrupts endolysosome function and stimulates lysosome biogenesis. MicrobiologyOpen. 2017;6:e416.

14. Reddy CA, Kao M. Value of acid metabolic products in identification of certain corynebacteria. J Clin Microbiol. 1978;7:428-33.

15. bioMérieux Brasil. Cartões VITEK ${ }^{\circledR} 2$ ID [cited 2018 Oct 25]. Available from: http://www.biomerieux.com.br/produto/ cartoes-vitekr-2-id

16. Kandi V, Palange P, Vaish R, Bhatti AB, Kale V, Kandi MR, et al. Emerging bacterial infection: identification and clinical significance of Kocuria species. Cureus. 2016;8:e731.
17. Vila J, Juiz P, Salas C, Almela M, de la Fuente CG, Zboromyrska $\mathrm{Y}$, et al. Identification of clinically relevant Corynebacterium spp., Arcanobacterium haemolyticum, and Rhodococcus equi by matrix-assisted laser desorption ionization-time of flight mass spectometry. J Clin Microbiol. 2012;50:1745-7.

18. Winn WC Jr, Chandler FW. Bacterial infections. In: Dail DH, Hammar SP, editors. Pulmonary pathology. $2^{\text {nd }}$ ed. New York: Springer; 1994. p.269.

19. World Health Organization. Global tuberculosis report 2017. Geneva: WHO; 2017.

20. Palmieri F, Girardi E, Pellicelli AM, Rianda A, Bordi E, Rizzi EB, et al. Pulmonary tuberculosis in HIV-infected patients presenting with normal chest radiograph and negative sputum smear. Infection. 2002;30:68-74. 